\title{
FORENSIC ART: 2D FACIAL COMPOSITE THROUGH IMAGE PROCESSING TECHNIQUES IN SRI LANKA
}

\author{
Sivaneasharajah L., ${ }^{1}$ Perera M.A.S., ${ }^{1}$ Jayasekara P.B., ${ }^{1}$ Perera J., ${ }^{2}$ Karunaratne D.D., ${ }^{1}$ \\ Sandaruwan K.D., ${ }^{1}$, Rajapakse R.N. ${ }^{1}$ \\ ${ }^{1}$ University of Colombo School of Computing \\ ${ }^{2}$ Department of Forensic Medicine and Toxicology, Faculty of Medicine, \\ University of Colombo, Sri Lanka
}

\begin{abstract}
INTRODUCTION

Forensic art is a technique used by law enforcement in order to identify a suspect towards apprehension or conviction of wanted persons and composite art is one branch. A "facial composite" is a graphical representation of a human face according to eyewitnesses' description. The failure rate of suspect identification through manual facial composite sketch was reportedly quite high in 2014 in Sri Lanka ${ }^{1}$. In addition, international software available for face identification may not be the best approach for Sri Lankan population.
\end{abstract}

\section{OBJECTIVE}

To develop an automated, image processing based computer software solution using 2D facial feature templates for suspect identification in Sri Lanka.

\section{STUDY DESIGN}

Facial feature templates were constructed by analyzing a dataset from Sri Lankan young adult population focusing on most occurring facial indices and commonly available facial feature shapes. Anthropometric proportion indices measurements and shape classification for eyes, nose, face, upper vermilion and lower vermilion of 140 undergraduates of both sexes between 20-25 age were identified and categorized according to BMI (Body Mass Index). Face Software
Development Kit library (Face SDK library) was used for index measurements and domain expert knowledge from aesthetic and forensic art fields were utilized in identifying facial feature shapes. These parameters were incorporated to construct 2D facial feature templates for major facial feature. Then these templates were transferred into the system to be used for composite construction purpose. Finally face visualization process will perform iteratively until the eyewitness is satisfied with the ultimate image. Evaluation of this solution with face pool technique and anthropometric index evaluation techniques obtained $70.19 \%$ accuracy and average of $84 \%$ respectively.

\section{CONCLUSION}

A significant accuracy level in composite images has been obtained by using anthropometric indices measurements. Hence, this computerized solution will significantly enhance the currently used manual facial composite procedure for suspect identification in Sri Lanka ensuring justice to the victim of crime.

Key words: Facial composite, anthropometric measurements, Facial indexes, Facial feature templates, Sri Lankan

Corresponding author: pererajean32@yahoo.com 
A "facial composite" is a graphical representation of a human face according to the eyewitnesses' description. In Sri Lanka forensic art for purposes of suspect identification is through manual hand drawing to obtain a facial composite. However, the failure rate of suspect identification through manual facial composite sketch was reportedly quite high in 2014 in Sri Lanka ${ }^{1}$. However, the failure rate of suspect identification through this manual facial composite sketch has been declared as $92.86 \%$ in the year 2014 in Sri Lanka ${ }^{1}$. This higher failure rate emphasizes the necessity of an immediate solution for the facial composite. It is also important to realize that incapability of identifying perpetrators of crime may leave the country in a precarious position as there is a seemingly rising number of crimes in the country. In order to overcome this situation, an automated, image processing based computer software solution has been introduced with 2D facial feature templates by incorporating both medically defined indices and aesthetic aspects ${ }^{5}$.

Even though there are numerous international software $^{2}$, these software are not the best suited to be adopted to Sri Lankan context as the facial feature templates which are available in these software may not be relevant for Sri Lankan population ${ }^{1}$.

Since Sri Lanka is at its infancy level in facial composite this research attempts to bridge the gap in order to find out the optimum way to automate this traditional procedure by incorporating the information technologies (image processing) and statistical analysis techniques and its related aspects. Thus as a first step, facial feature templates need to be constructed by analyzing a dataset from Sri Lankan population.
In early studies it was proven that the ability of assessing facial feature appearance has been achieved by anthropometric proportion indices and this has been used for a number of forensic practices ${ }^{3}$. Hence, when constructing the facial feature templates this research focused on two major parameters known as most occurring indices andcommonly available facial feature shapes. In order to find the relative measurements for these parameters, two sub researches on facial anthropometric indices and shape classification were carried out targeting the local population. Therefore in the first phase of the research, aforementioned sub researches were conducted with 140 undergraduate students (both male and female) who are aged 20-25 to find out the anthropometric proportion indices measurements and the shape classification for eyes, nose, face, upper vermilion and lower vermilion for Sri Lankans. Moreover the entire data set was categorized based on the weight categories according to BMI (Body Mass Index) values as BMI can be used to screen different weight categories.

The index measurements were measured with the use of Face SDK library as it is comparatively more reliable and accurate over manual measuring techniques whereas the domain expert's knowledge from the aesthetic and forensic art field were utilized in identifying the facial feature shapes. Afterwards by incorporating these parameters as shown in Fig. 1, 2D facial feature templates for face, eye, nose and mouth were created targeting Sri Lankan people and eventually these templates were then transferred into the system which can be used for the composite construction purpose. 


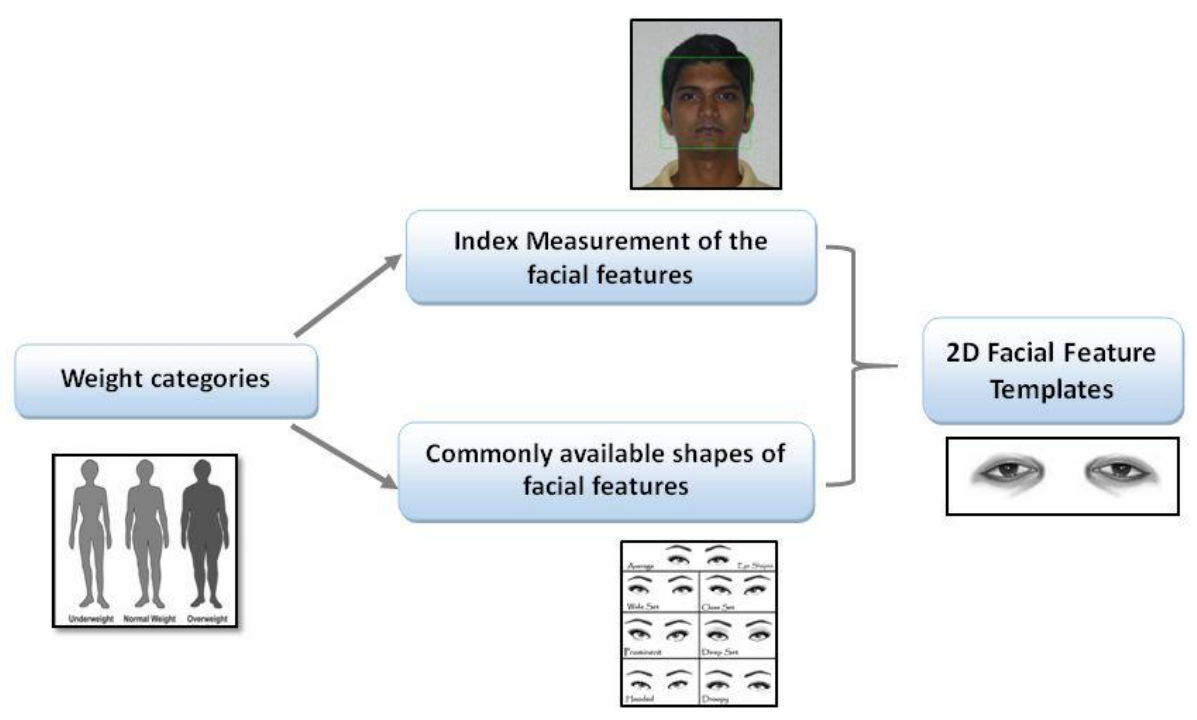

Figure 1: Facial feature template creation process by incorporating anthropometric indices and commonly available shapes

In addition, this system enables filtering the most possible feature templates based on the eyewitness description given with the age, height, weight and other criteria. Besides, this research study has identified the template positioning of each facial feature by analyzing the distance between the facial features and incorporating the standard divine proportions $^{4}$ which is suitable for the Sri Lankan context. Finally, the face visualization process will perform iteratively until the eyewitness is satisfied with the ultimate composite image.

This solution has been evaluated ${ }^{5}$ with face pool and anthropometric index evaluation techniques where the face pool evaluation technique has obtained $70.19 \%$ accuracy. On the other hand the anthropometric index evaluation method obtained average of $84 \%$ of out of the population who gave a response regarding the matching of the faces. This shows the significance of the accuracy level in composite images, when it uses the anthropometric indices measurements. Hence, this fully implemented computerized solution will significantly enhance the current manual facial composite procedure and eventually this proposed solution will benefit personnel to provide services to the criminal justice process such as law enforcement officers, legal counsel and ensure that victimized person has quick access to justice.

\section{ACKNOWLEDGEMENTS}

Authors gratefully acknowledge the advisor Mr. Winnie Hettigoda, Senior lecturer of University of the Visual \& Performing Arts and Senior Superintendent of Police Mr. Priyantha Jayakody, Director of Criminal Record Division in Sri Lanka who helped us in gaining the relevant domain knowledge. 


\section{REFERENCES}

1. Personal communication, (2015) Manual Process of Suspect Identification through Facial Composite in Sri Lanka, Interviewee, Criminal records division of Sri Lanka police.

2. Frowd, C., McQuiston-Surrett, D., Anandaciva, S., Ireland, C. and Hancock, P. (2007) An Evaluation of US Systems for Facial Composite Production. Ergonomics, 50, 1987-1998. $\quad$ http://dx.doi.org/ 10.1080/00140130701523611

3. Rajapakse, R., Madugalla, A., Amarasinghe, I., Padmathilake, V., Dharmaratne, A., Sandaruwan, D. and Vidanapathirana, M. Facial Muscle Anatomy Based Approach for Forensic Facial Reconstruction in Sri Lanka. International Conference on Advances in ICT for Emerging Regions (ICTer)2012, Colombo, 27-35.

http://dx.doi.org/10.1109/icter.2012.6422828.
4. Y. Jefferson, "Skeletal Types: key to unraveling the mystery of facial beauty and its biological significance," Journal of General Orthodontics, June 1996; vol. 7, pp. 10-12.

5. Jayasekara PB, Sivaneasharajah L, Perera MAS, Perera J, Karunaratne DD, Sandaruwan KD, Rajapakse RN. Facial Index Based 2D Facial Composite Process for Forensic Investigation in Sri Lanka. Journal of Forensic Medicine and Anatomy Research, (2016);4:7 - 16 